\title{
Consumer's Opinion on a Pharmacist's Role in Nutritional Counseling
}

Megan Meier, Doctor of Pharmacy student; Reshmi L. Singh, BPharm, MS, PhD; Baskaran Thyagarajan, MPharm, PhD

School of Pharmacy, University of Wyoming

\begin{abstract}
Background: Nutrition has become an increasing health concern. From fad diets to exercise programs, the consumer seems to be consulting multiple resources that all provide a different answer in the process of obtaining optimal health. Easily accessible pharmacists may be the sources to answer crucial nutritional issues facing society today.

Methods: Using the results of a previous study about food perceptions among lean and non-lean populations, interview questions were created to determine the populations' nutrition and pharmacist's role knowledge. Nutrition knowledge among lean vs. non-lean consumers was assessed by determining experience with pharmacist nutritional counseling, how experience perceptions differed, and how these perceptions can shape a pharmacist's role. Audio recorded interviews were conducted in a rural setting. Seventy-two English speaking, ambulatory, lean (BMI $\leq 24)$ and non-lean $(B M I \geq 25)$ residents between the ages $25-71$ years participated in the study in a small western United States town.

Results: $26.3 \%$ of consumers obtained nutrition information from the internet; however, the internet and healthcare providers were considered the most trustworthy. When asked about the pharmacist's role in nutrition counseling, $32.7 \%$ believed that pharmacists were not a reliable source based on education background. Another 10.2\% thought pharmacists were too busy for counseling.

Discussion: This study indicates the consumers' knowledge on pharmacists' education, willingness to seek out nutrition knowledge, and a pharmacist's ability to provide nutrition counseling can differ between lean and non-lean consumers. Lean consumers tend to have more interaction with a pharmacist whereas non-lean consumers do not.

Conclusion: As a profession, pharmacists should educate consumers on reliable nutrition resources, a pharmacist's education, and how they use their role to provide consumers with nutrition knowledge.
\end{abstract}

Keywords: nutrition, counseling, pharmacy education, public health, patient education

\section{BACKGROUND}

Nutrient counseling and the pharmacist's role in it are of significant interest among researchers. People have tried a variety of diets and exercise regimens; however, there has never been a nutritional strategy for every individual to maintain optimum health. When homecare administrators were questioned about their perception on the number of malnourished patients, $39.6 \%$ of those surveyed agreed that most patients are malnourished. ${ }^{1}$ Pharmacists receive extensive training on a variety of supplements that enhance a person's overall health; however, many consumers are not aware of a pharmacist's nutrition knowledge. Therefore, consumers do not seek counseling and education about their diet and nutrition from a pharmacist. A survey by Rosenthal and colleague survey reported that 100 community pharmacists in Canada thought consumers viewed them as "dispensers of medication" instead of patient-centered. ${ }^{2}$ Only $12.7 \%$ of pharmacists are employed in settings requiring nutrition counseling, such as homecare. ${ }^{1}$ This creates an obstacle in healthcare since consumers are lacking education about nutritional supplements they take.

Corresponding author: Megan Meier, Doctor of Pharmacy student; 1000 E University Ave, Laramie, WY 82071

Phone: 719-433-5647

Email:mmeier6@uwyo.edu
Evidence from previous studies shows a lack of nutritional counseling and knowledge about a pharmacist's role in the general population. ${ }^{4,5,6,7,8}$ Often an overweight patient will receive more counseling than a smoking patient. ${ }^{4}$ While pharmacists do possess the nutritional counseling credentials, consumers often do not perceive a pharmacist as an expert on the subject nor do they perceive the pharmacist having the time to counsel on the matter. Consumers identified time, space, education, and insurance reimbursement programs as barriers to a pharmacist providing adequate nutrition counseling. ${ }^{5} \mathrm{~A}$ Scottish study supported this by identifying lack of awareness, privacy concerns, lack of comfort talking to pharmacy staff about weight management, and the lack of a pharmacist's knowledge as key factors in preventing the public in seeking advice from a pharmacist. ${ }^{6,7}$ Furthermore, an English study asked women about their perception of pharmacists counseling on weight loss and while most were comfortable asking pharmacists, only 39\% ranked pharmacists over physicians, dieticians, and exercise consultants as experts on the subject. ${ }^{5}$ Another study in Australia showed that respondents never considered asking pharmacists about weight management services because they distrust pharmacists' motives, expertise, and view a pharmacist as too busy to provide those services. ${ }^{5,7}$ Clearly a knowledge gap of a pharmacist's perceived responsibilities and true responsibilities exists. Perepelkin's study implied that many respondents report a "poor/fair" understanding of a pharmacist's education, what to expect 
from counseling, and a pharmacist's scope of practice. ${ }^{8}$ Consumers are not aware of the education involved in becoming a pharmacist or the services a pharmacist can provide. ${ }^{4,5,6,7,8}$

Pharmacists are trained in a variety of ways to meet consumer's nutritional needs. ${ }^{9,10,11,12,13,14}$ Pharmacists have received education on multiple disease states, risk factors, prevention of disease, management of chronic diseases, and physical assessments. ${ }^{9}$ While pharmacists are great resources on disease management and medications, they also are trained in communicating with patients. All pharmacy schools teach motivational and behavioral interviewing strategies in order to help pharmacists individualize treatment plans. ${ }^{10}$ Moreover, pharmacists are educated in nutrition. Nutrition textbooks used by pharmacy schools address the human diet, counseling advice, and dietary supplements. ${ }^{11}$ Furthermore, pharmacy school curriculum provides education on lifestyle monitoring including the DASH diet and balancing vitamin levels. ${ }^{10}$ Programs to advance a pharmacist's ability to manage diabetes specific nutrition have been developed as part of advanced pharmacy practice experiences (APPE) completed during the final year of pharmacy school. ${ }^{12}$ At the University of Illinois, a nutrition class was offered to second and third year pharmacy students. ${ }^{13}$ After the class, most students agreed they had enhanced their nutrition knowledge by increasing application of research into clinical practice and understanding how culture effected a patient's food choices. ${ }^{14}$ Even in undergraduate education, there is a movement to educate future pharmacists on nutrition. An Australian pharmacy undergraduate program, created a course to teach nutrition labeling and counseling in accordance with the Australian Guide to Healthy Eating. ${ }^{14}$ Additionally, pharmacists have parenteral nutrition education. As many as $74 \%$ of pharmacists have made errors when administering medications via feeding tube. ${ }^{12}$ A pharmacist's role during parenteral nutrition administration is to prevent drug interactions, malnourishment, and electrolyte imbalances. ${ }^{15}$ Aside from parenteral nutrition, a pharmacist can also provide nutrition counseling related to diet. A relatively new nutrition-related specialty is sports pharmacy. Pharmacists have a vast knowledge of supplements that are commonly used in sports; however, only $52 \%$ of athletes said they would talk to a pharmacist about sports supplements. ${ }^{16}$ A pharmacist's training is far beyond the scope of just medications. ${ }^{9,10,11,12,13,14}$

This study's primary objective was to determine the sources used by consumers to obtain their nutrition information and what they deem a pharmacist's role in nutrition is. Secondary objectives included determining the amount of interaction and experience the average consumer has with a pharmacist and the consumers' perception of a pharmacist's job and education. The study also considered if there was a difference between how a lean or non-lean consumer responded to questions about experiences with nutrition counseling and perceptions about a pharmacist's role.

\section{METHODS}

Drawing conclusions from Menghini et al.' $\mathrm{s}^{19}$ mixed method analysis, a qualitative research design was implemented to gain more insight on how consumers obtain nutrition knowledge, as well as their perception of the pharmacist's role in providing nutrition counseling. Subjects granted written informed consent and all subjects' rights are protected by the Human Subjects Review Board. Open ended questions regarding use of nutritional resources, supplement use, and healthcare professional nutrition counseling experience were asked to each participant during an audio-recorded interview.

To recruit consumers for the study, it was advertised on a university campus and around the university town. Seventytwo consumers responded to interview questions. These consumers reported their age, gender, marital status, education, weight, and height. Weight and height were used to calculate Body Mass Index (BMI) in order to determine if the consumer was lean or non-lean. Lean individuals were classified as a $\mathrm{BMI} \leq 24$, while non-lean individuals had a $\mathrm{BMI} \geq 25$. Participant demographics are displayed in Table 1.

Table 1: Consumer Selection

\begin{tabular}{|l|l|l|}
\hline Demographic & Numbers $(\mathbf{n}=)$ \\
\hline Gender & Males & Females \\
& $\mathrm{n}=19$ & $\mathrm{n}=53$ \\
\hline Lean & Yes & No \\
& $\mathrm{n}=36$ & $\mathrm{n}=36$ \\
\hline Age & $<45$ years & $\geq 45$ years \\
& $\mathrm{n}=37$ & $\mathrm{n}=35$ \\
\hline
\end{tabular}


A one-on-one interview addressing seven open-ended questions was recorded. Data collection consisted of openended questions and consumers' demographic information. Questions were asked regarding where consumers found nutritional information, what resources consumers considered trustworthy, experience obtaining nutritional advice, willingness to seek dietary advice from a pharmacist, importance of receiving medication counseling, experience with dietary supplements, and a credibility ranking of various healthcare professionals providing nutritional counseling (Figure A.1). Textual responses were the product of qualitative data collection and responses to designated open-ended items. Two research faculty conducted the interviews, which were digitally recorded. Responses were recorded verbatim.

A horizontal approach was used during the first data analysis. Since this was an exploratory study, predecided theory was not used. Instead, the use of qualitative description was appropriate to stay "data-near" to the consumer descriptions of their experiences and avoid unnecessary interpretation from the authors. Researchers began by reading each survey response and taking notes. Then clusters for each question were created based on common themes found in responses. For instance, the common themes when asked why a consumer would or would not consult a pharmacist for nutritional counseling were: yes, if a medication is involved; yes, because of personal interactions with a pharmacist; yes, pharmacists have proper training; no, a pharmacist is too busy; no, a pharmacist does not have proper training; no, because of no personal relationship with a pharmacist; never thought about it; and depends on the situation. The final step in the analysis consisted of comparing lean and non-lean consumers' response to nutritional knowledge and their perception of pharmacists providing nutritional information. Comparisons were made between how lean and non-lean individuals responded. Themes were created to address what resources respondents used to inform themselves about nutrition and to determine the respondents' level of knowledge surrounding a pharmacist's education, role, and responsibilities regarding nutrition. When making comparisons and grouping common themes, researchers quantified the data by calculating percentages for ease of communicating results. Faculty researchers coded separately. Codes were discussed at a later time to resolve any disagreements and ensure rigor was maintained. Since interpretation was not part of this methodology, data near qualitative analysis resulted in consensus of results.

The authors are all from pharmacy background. However, each comes from a different positionality in that the first author is a student who will be a clinical pharmacist upon graduation and was interested in not only seeing the perception of consumers regarding practicing pharmacists' role in nutrition counseling, but also how these roles could differ in different clinical settings. In addition, the student author did reflect on her own nutrition education experience during the PharmD program. Second author comes to this research from a social pharmacy and qualitative research perspective to understand consumer experiences and perceptions of pharmacists' role in nutrition counseling. In addition, being a pharmacy educator, the second author also was interested in learning the implications for nutrition education in the PharmD curriculum. Similarly, third author had the commonality of being a pharmacy educator and in addition being a basic scientist. This author was interested in the research from a clinical and translational lens of understanding lean and non-lean consumer differences. Overall, the different lens provided further credibility once consensus on themes was obtained.

\section{RESULTS}

After interviews concluded and data was analyzed, three main themes were found. These three findings include: consumers seek nutrition knowledge through online resources, lack of interaction with pharmacists regarding nutrition is high among consumers, and misperceptions deter consumers from consulting pharmacists about nutrition. The findings are recorded below along with figures and tables as representation.

\section{Consumers seek nutrition knowledge through online resources}

Our study suggested that most consumers consult the internet for nutritional information. Of the $41 \%$ that consulted the internet $42 \%$ are lean. $20 \%$ of study participants consulted healthcare professionals and of this $58 \%$ are lean. $8.5 \%$ of study participants used family as a resource and $40 \%$ of those were lean. A small $5.1 \%$ of study participants used books and of those $33.3 \%$ were lean. All $3 \%$ of consumers who used their personal experience are lean. And $20 \%$ reported using a mixture of the listed resources to find nutritional information and $58.3 \%$ of those were lean. See Figure 1 and Table 2. 


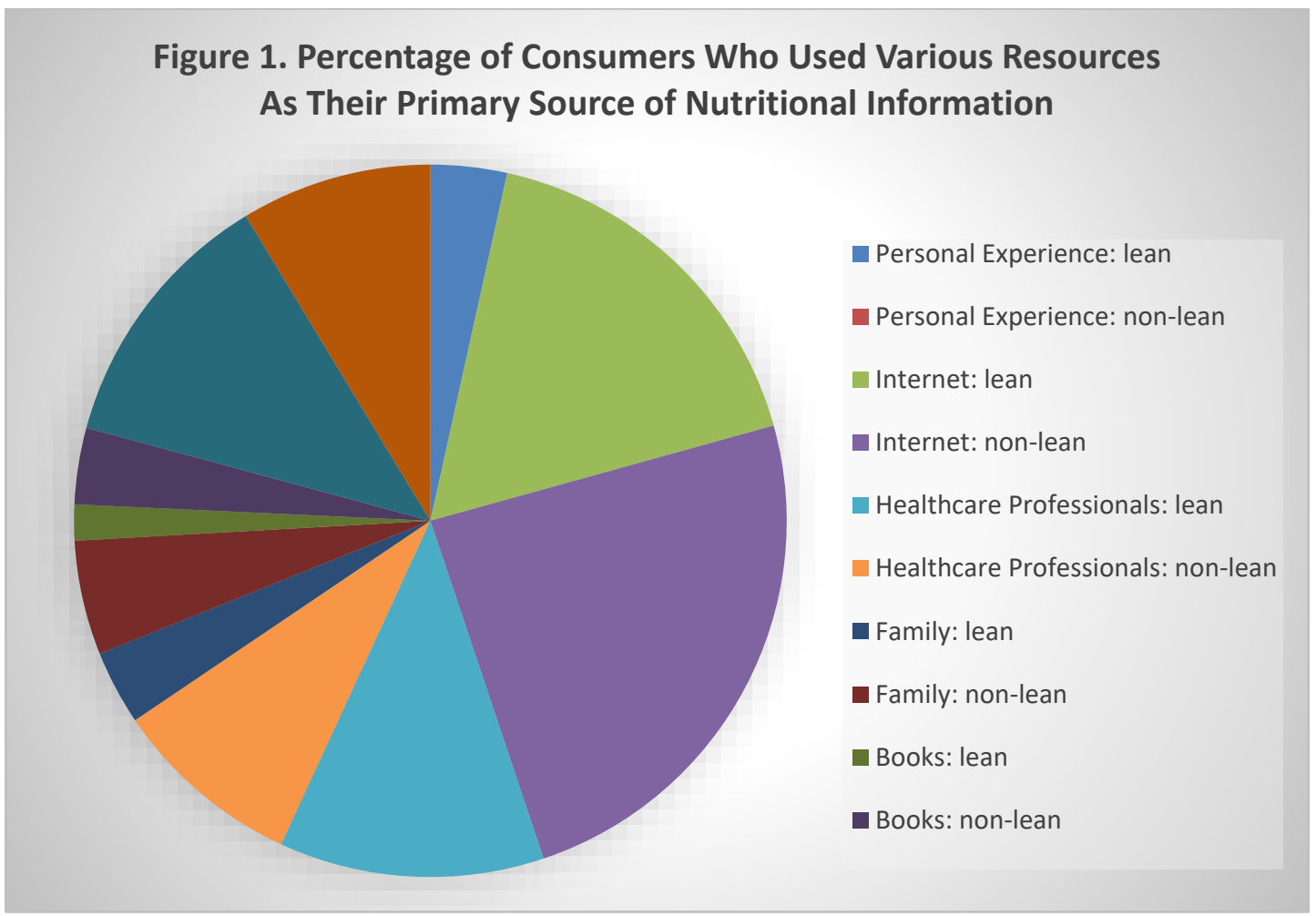

Table 2: Percentage of consumers who used various resources as their primary source of nutritional information.

\begin{tabular}{|l|l|}
\hline Subject Number & Quotation \\
\hline 38 & "Dr. Google" \\
\hline 1 & "What most people probably do is just go to the Internet and hope for the best" \\
\hline 1 & "Definitely use the internet some. I'm cautious of what's out there" \\
\hline
\end{tabular}

Lack of interaction with pharmacists regarding nutrition is high among consumers

Consumers were asked if they had ever purchased supplements and if they consulted a pharmacist before, during, or after their purchase. $14 \%$ of consumers had purchased supplements and a pharmacist was consulted. $57 \%$ of these consumers were non- lean. An overwhelming $65 \%$ of consumers had purchased supplements and not consulted a pharmacist. $58 \%$ of these consumers were non-lean. $24 \%$ of consumers had never purchased supplements. $58 \%$ of these consumers were lean. See Figure 2 and Table 3. 


\section{Figure 2. Percentage of lean vs. non-lean consumers who} have never consulted a pharmacist about supplements

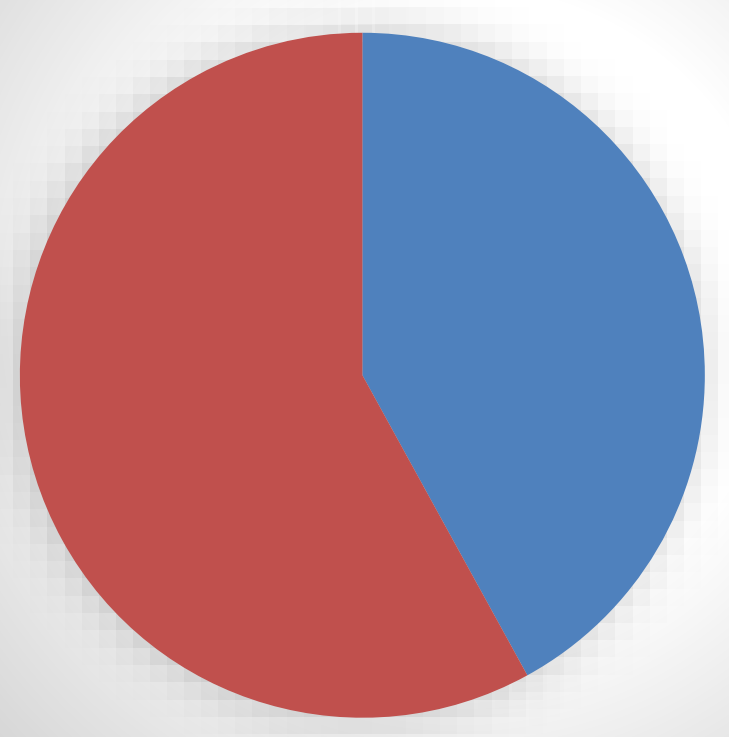

Lean $\square$ Non Lean

Table 3: Quotations from consumers regarding trustworthy nutrition resources.

\begin{tabular}{|l|l|}
\hline Subject & Quote \\
\hline 62 & $\begin{array}{l}\text { "I never thought about it before"-when consumer asked if he views pharmacist as a } \\
\text { trustworthy nutrition resource }\end{array}$ \\
\hline 66 & $\begin{array}{l}\text { "I have asked them about over-the-counter medications and how it will affect me and my } \\
\text { kids. I don't think it really would occur to me to ask them about food" }\end{array}$ \\
\hline 72 & $\begin{array}{l}\text { "It would be good to know what foods to stay away from, if it's going to cause some } \\
\text { issues between medication, and what it's supposed to be doing" }\end{array}$ \\
\hline
\end{tabular}

Misperceptions deter consumers from consulting pharmacists about nutrition

Consumers are willing and not willing to consult a pharmacist about nutrition. Each group has its own reasons for as to why or why not they will consult a pharmacist. Of the consumers who never consulted a pharmacist $33 \%$ believed pharmacists do not have the proper training and of this segment $50 \%$ are lean. $10 \%$ believed pharmacists are too busy and $80 \%$ of this segment is non-lean. Another $4.1 \%$ responded that they would not consult a pharmacist because they have no relationship with the pharmacist. All of these respondents were lean.
$25 \%$ of consumers never thought about consulting a pharmacist and $50 \%$ of this population is lean. Of the population who consulted pharmacists, $12 \%$ said they would consult a pharmacist because of their training with $50 \%$ of those being lean, $6 \%$ would consult pharmacists because of personal interactions with all of the segment being lean, and another $6 \%$ would consult pharmacists if medications were involved with all of this segment being non-lean. $4.1 \%$ of study participants said consulting a pharmacist about nutrition would depend on the situation with all of that segment being lean. See Figure 3 and Table 4. 


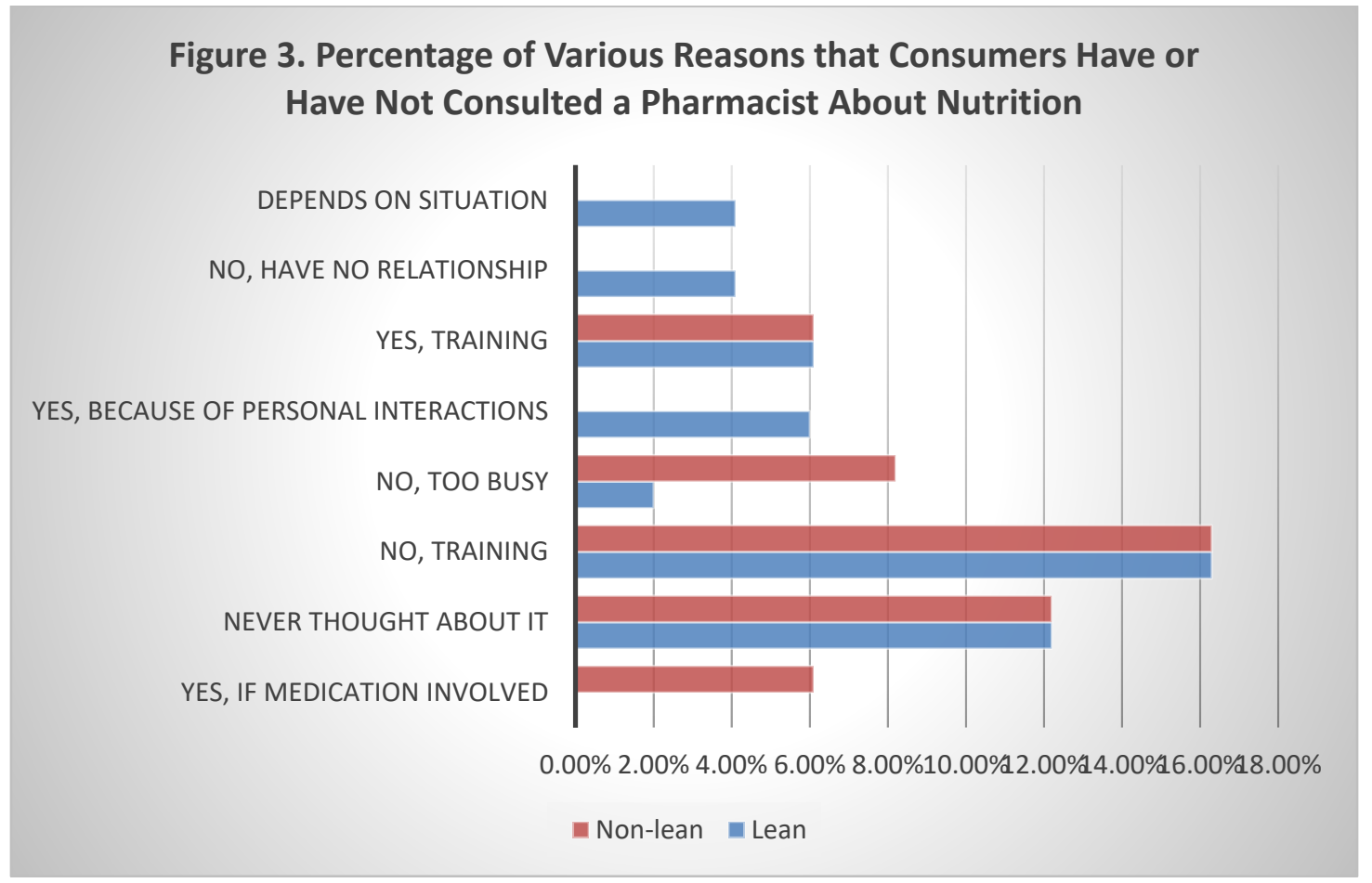

Table 4: Quotations from consumers about why they have or have not consulted a pharmacist about nutrition.

\begin{tabular}{|l|l|}
\hline Subject & Quotation \\
\hline 62 & "They always seem so busy" \\
\hline 68 & "I first see them as just dealing with drugs, chemicals" \\
\hline
\end{tabular}

\section{DISCUSSION}

Consumers' outlook on pharmacists' education, a pharmacist's ability to provide nutrition, and willingness to seek out nutrition knowledge can differ between lean and non-lean consumers. Lean consumers are those with a BMI $\leq 24$ and non-lean consumers are those with a $\mathrm{BMI} \geq 25$. Most consumers received their nutrition knowledge from the internet and of those reporting use of the internet most were non-lean. Largely, consumers do not depend solely on healthcare professionals for nutrition and health-related knowledge. However, a higher percentage of non-lean individuals sought information from the internet. Lean individuals obtained information from healthcare professionals other than a pharmacist. The data suggest that nutrition information from a trained healthcare professional is more reliable and assist consumers in reaching a healthy weight. All other differences between lean and nonlean consumers attaining nutritional knowledge did not differ greatly.
Regarding trustworthy resources, consumers considered healthcare professionals, the internet, and academic researchers to be the most trustworthy. The most popular resources are the ones consumers considered the most trustworthy suggesting consumers believed they were receiving valid information. $74.9 \%$ of consumers believed dieticians were the most trustworthy because of their profession's specific education. Moreover, consumers do not seek out other resources, such as pharmacists, because they are content with their current resources and believe they are optimizing their health without consulting a pharmacist.

The data suggests $42 \%$ of lean and $58 \%$ of non-lean consumers have never interacted with pharmacists about nutrition or supplements. Non-lean consumers may not receive counseling because a stereotype exists. A pharmacist may assume a nonlean individual may not want to learn about nutritional supplements or physical assessments. Another explanation is that non-lean individuals are shy and self-conscious about their weight leading them to not ask questions nor seek nutritional 
counseling. On the other hand, lean consumers have a higher percentage of interactions with pharmacists and other health professionals because they are proactive about their health and have sought health information to maintain a lean frame. Although, the portion of lean consumers not interacting with pharmacists could be attributed to the population not possessing a need to consult a healthcare professional. The same percentage of non-lean consumers consulting pharmacists was equal to the percentage of non-lean consumers not consulting a pharmacist during a supplement purchase. Therefore, being non-lean does not dictate if a person consulted a pharmacist or not. This could be a result of the general population not being educated on a pharmacist's role in healthcare. When considering gender, no significant differences were found between male and female participants, as the study's population was mostly females.

Consumers do not interact with pharmacists regarding nutrition as much as other health professionals. This can be attributed to $33 \%$ of consumers regarded pharmacists as professionals lacking proper training to consult on nutrition, $25 \%$ of consumers have never considered consulting a pharmacist, and another $10 \%$ of consumers viewed pharmacists as too busy to offer counseling. However, some consumers believed pharmacists should be consulted about nutrition attributing it to their training. If a consumer-pharmacist relationship is formed this perception could change, as a consumer would feel they are confiding in a trusted individual who would be willing to take the time to answer nutritional questions. There is a discrepancy in the public's knowledge about a pharmacist's education. Pharmacists' roles are evolving by collaborating with other healthcare professionals to provide the most comprehensive care in nutrition. ${ }^{5,17,18}$ Healthcare professionals around the world are working together to improve treatment of non-communicable diseases, which was seen in the WHO's Global Action Plan for the Prevention and Control of Noncommunicable Diseases. ${ }^{5}$ This plan included:

1. increasing physical activity

2. decreasing risk of cardiovascular disease, cancer, diabetes, and respiratory diseases

3. decrease the number of diabetes and obesity cases

Measures to meet the WHO's plan are already in effect at Auburn University. Auburn's pharmacy faculty and students take a comprehensive patient social and medical history as well as a physical assessment in order to plan a patient's nutrition therapy. The Lifestyle Challenge Program at a New York outpatient clinic had a nutrition expert physician, pharmacist, and behavioral psychologist seek to make diet and exercise interventions for patients with a BMI over $27 \mathrm{~kg} / \mathrm{m}^{2}$. Participants lost a total of $4.8 \%$ body weight suggesting collaborative healthcare practice is beneficial. ${ }^{5}$ These programs have helped patients lose weight; however, for patients to maintain their target weight pharmacists need more education on counseling and monitoring. In a 2007 survey, fewer than $25 \%$ of pharmacy schools offer lifestyle modification courses. ${ }^{17}$ Students were asked how they felt about providing nutritional counseling prior to the survey and after they were instructed to conduct a meal plan for diabetic patients. ${ }^{18}$ All respondents thought that counseling should be provided on lifestyle modifications, but only $54 \%$ felt they had received sufficient education to satisfy this need. According to one study, upon receiving additional education, students and preceptors reported improvement in the student pharmacist's ability to provide individualized nutrition counseling. ${ }^{18}$ Based on these and other findings, the Accreditation Council for Pharmacy Education (ACPE) in 2016 required non-pharmacological counseling to be included in curriculum. ${ }^{17}$ With interprofessional and nutrition education, pharmacists can make an impact in improving nutrition counseling services. ${ }^{5,17,18}$

As a profession, pharmacists should actively educate the public on their education, roles, and responsibilities within healthcare. Consumers who thought pharmacists are too busy to counsel do not recognize that part of a pharmacist's role is to consult on medications and any health-related issues. Further studies should display to the public that pharmacists are trained to counsel and do not only dispense medications. These future studies can create methods by which this can be accomplished such as support of counseling activities through payment for services with MTM, CMM, CCM, TCM, ADA or AADE.

A limitation of this study is the unequal amount of male and female participants. There were largely more females in the study. Another limitation is the sole focus on the qualitative aspect of data. Other than percentages calculated, no results have been quantified. This study illustrates concepts, but for any statistics a larger study will need to be done.

\section{CONCLUSION}

Some social factors did influence consumer responses to pharmacists providing nutritional counseling, while some did not. Gender did not significantly influence consumers' responses; however, there were differences among the nonlean and lean populations. Non-lean consumers were more likely to not consult a pharmacist about nutrition. In general, the study's population consulted the internet and healthcare professionals most about nutrition. When consumers were asked about why they do not consult pharmacists about nutrition, most reported they did not believe pharmacists have the proper training and are too busy to provide a consult. This data suggested that consumers are not educated on a pharmacist's training; therefore, they never considered to even consult a pharmacist about nutrition.

This study provided a qualitative analysis of consumers' opinions about attaining nutritional information and 
pharmacists providing nutritional counseling. Further studies could provide quantitative data to show a statistical significance between males and females as well as varying age groups. Implications of this study involve educating the public on pharmacists' education and role in providing nutrition counseling. Further studies should assess consumers' level of understanding regarding the pharmacist's education and role in creating as well as providing a nutritional counseling program to all populations including pediatrics, geriatrics, and athletes. Additional research could reveal a pivotal role for pharmacists in providing nutritional education to subpopulations. Pharmacists should be educated to use motivational interviewing when talking with patients about nutrition. Pharmacist's education should also encourage pharmacists to advocate for adequate time to discuss nutrition with patients. Pharmacists need to make counseling a priority as many consumers do not receive adequate information regarding medications or supplements at regular physician visits.

This study shows where a consumer receives nutritional information and their perception of a pharmacist as a nutrition counselor. Differences between lean and non-lean consumers' responses were also considered. In the future, the study could focus more on analyzing ways to educate the public on a pharmacist's role in nutrition to bridge the knowledge gap that exists.

Acknowledgments: The authors of this paper would like to thank all the study participants for their time to answer the study questions. We also would like to extend gratitude to Dr. Lawrence Staubach for his pharmaceutical knowledge and assisting with data collection. Special thanks to Mr. Mark Menghini, Research student at the Molecular Signaling Laboratory University of Wyoming School of Pharmacy, for his kind review and suggestions. Another special thanks to Justine Jayne, PharmD for her help with recruiting participants, transcription of interviews and data analysis.

Funding: This work was supported by the Mountain West Clinical Translational Research - Infrastructure Network with a grant from National Institute of General Medical Sciences of the National Institutes of Health under Award Number IU54GM104944. Content was the responsibility of the authors and does not represent the official views of the National Institute of Health.

Conflicts of interest: We declare no conflicts of interest or financial interests that the authors or members of their immediate families have in any product or service discussed in the manuscript, including grants (pending or received), employment, gifts, stock holdings or options, honoraria, consultancies, expert testimony, patents and royalties.
Treatment of Human Subjects: IRB review/approval required and obtained.

\section{Author contributions: \\ Megan Meier: Formal analysis, Resources, Writing-Original Draft, Writing-Review \& Editing, Visualization \\ Reshmi L Singh: Conceptualization, Methodology, Validation, Investigation, Writing-Review \& Editing, Supervision, Project Administration \\ Baskaran Thyagarajan: Validation, Writing- Review \& Editing, Visualization, Supervision, Project Administration}

\section{REFERENCES}

1. Schiller MR, Arensberg MB, Kantor B. Administrators' perceptions of nutrition services in home health care agencies. J Am Diet Assoc. 1998;98(1):56-61.

2. Rosenthal MM, Breault RR, Austin Z, Tsuyuki RT. Pharmacists' self-perception of their professional role: insights into community pharmacy culture. 2011;51:363-367.

3. Hickey E, DiPiro J, Romanelli F. Prospective health professions students' misperceptions about pharmacists. Am J Pharm Educa. 2019;83:1175-1178.

4. Heaton PC, Frede SM. Patients' need for more counseling on diet, exercise, and smoking cessation: results from the national ambulatory medical care survey. J Am Pharm Assoc. 2006; 46:364-369.

5. Jordan M, Harmon J. Pharmacist interventions for obesity: improving treatment adherence and patient outcomes. Integr Pharm Res Pract. 2015;4:79-89.

6. Weidmann AE, Cunningham S, Gray G, et al. Views of the scottish general public on community pharmacy weight management services: international implications. Int J Clin Pharm. 2012;34: 389-397.

7. Borralho J, Gregório J. "Each one has their own role": Exploratory study on consumers' perceptions about nutritionists services provided in community pharmacies. Res Social Adm Pharm. 2020;S15517411:30331-30334.

8. Perepelkin J. Abramovic M. Public education campaigns to transform perceptions of pharmacists: are they worth the investment? Health Commun;31:833-844.

9. Charuel A, Prevost V. Nutritional counseling in community pharmacies within the framework of the french national nutrition and health program. Ann Pharm Fr. 2014;72:337-347.

10. Earl G, Henstenburg JA. Dietary approaches to hypertension: a call to pharmacists to promote lifestyle changes. J Am Pharm Assoc. 2012;52:637645.

11. Wolinsky, I, Williams L. Nutrition in pharmacy practice. Am J Pharm Educa. 2002;11(2):Article 58. 
12. Herring $M$, Beckett $E$, Stanton-Robinson $C$, Witry $M$. What do l eat? impact of an interactive teaching method for improving pharmacy students' diabetes nutrition knowledge and comfort in providing nutrition counseling. Curr Pharm Teach Learn. 2018;10:918-924.

13. Chang L, Popovich N, Iramaneerat C, et al. A clinical nutrition course to improve pharmacy students' skills and confidence in counseling patients. Am J Pharm Educa. 2008;72(3):Article 66.

14. Pearce K, Cross G. A 4-week nutrition and therapeutics course in an undergraduate pharmacy program. Am J Pharm Educ. 2013;77(7):Article 154.

15. Ekincioğlu $A B$, Demirkan K. Clinical nutrition and drug interactions. Ulus Cerrahi Derg. 2013; 29:177-186.

16. Howard M, DiDonato K, Janovick D, et. al. Perspectives of athletes and pharmacists on pharmacist-provided sports supplement counseling: an exploratory study. J Am Pharm Assoc. 2018;58:S30-S36.
17. Herring MS, Beckett EA, Stanton-Robinson CA, Witry MJ. What do I eat? Impact of an interaction teaching method for improving pharmacy students' diabetes nutrition knowledge and comfort in providing nutrition counseling. Curr Pharm Teach Learn. 2018;10:918-924.

18. Taylor SR, DeGeeter M, Wilson JA, Leadon KI, Rodgers PT. Preceptor perceptions of fourth year student pharmacists' abilities regarding patient counseling on therapeutic lifestyle changes. Curr Pharm Teach Learn. 2016;8:353-358.

19. Menghini M, Singh $R$, Thyagarajan B. Understanding food preferences and their connection to health perception among lean and non-lean populations in a rural state. Innovations in Pharmacy. 2020;11(4):17.

\section{APPENDIX}

Pharmacists and Nutritional Counseling Questions

1. Where do you normally get your information regarding food choices and nutrition? Friends or family? Internet, books, or magazines? Other?

2. Which sources do you consider most trustworthy? Why?

3. Please describe your experience seeking nutritional advice from healthcare professionals? Can you give a specific example? What specifically was the context of the question?

4. Is a pharmacist someone from whom you might seek dietary or nutritional advice? Why or why not?

5. Please describe the importance of being able to get nutritional counseling when purchasing prescription medications, OTC medications or nutritional supplements in your local community pharmacy?

6. Please rank order your perceived credibility of the following health professionals in providing nutritional counseling: nurses, pharmacists, MDs, and dieticians.

7. What is your experience with dietary supplements? Was a pharmacist involved in your decision making? Provide an example.

Figure A.1. Interview Questions 